\title{
Illegitimacy and sibship assignments in oil palm (Elaeis guineensis Jacq.) half-sib families using single locus DNA microsatellite markers
}

\begin{abstract}
Oil palm breeding has been progressing very well in Southeast Asia, especially in Malaysia and Indonesia. Despite this progress, there are still problems due to the difficulty of controlled crossing in oil palm. Contaminated/illegitimate progeny has appeared in some breeding programs; late and failure of detection by the traditional method causes a waste of time and labor. The use of molecular markers improves the integrity of breeding programs in perennial crops such as oil palm. Four half-sib families with a total of 200 progeny were used in this study. Thirty polymorphic single locus DNA microsatellites markers were typed to identify the illegitimate individuals and to obtain the correct parental and progeny assignments by using the CERVUS and COLONY programs. Three illegitimate palms (1.5 $\%$ ) were found, and 16 loci proved to be sufficient for sibship assignments without parental genotypes by using the COLONY program. The pairwise-likelihood score (PLS) method was better for half-sib family assignments than the full likelihood (FL) method.
\end{abstract}

Keyword: Half-sib; Illegitimacy; Microsatellite; Oil palm; Sibship assignment 